\title{
Clinicopathological Features of Small Bowel Tumors Diagnosed by Video Capsule Endoscopy and Balloon-Assisted Enteroscopy: A Single Center Experience
}

\author{
Ah Young Yoo, Beom Jae Lee, Won Shik Kim, Seong Min Kim, Seung Han Kim, Moon Kyung Joo, Hyo Jung Kim and Jong-Jae Park \\ Division of Gastroenterology, Department of Internal Medicine, Korea University Guro Hospital, Korea University, College of Medicine, \\ Seoul, Korea
}

Background/Aims: Small bowel malignancies often present a diagnostic challenge due to their relative rarity and nonspecific clinical symptoms. However, technical developments in endoscopic instruments, including video capsule endoscopy (VCE) and enteroscopy, have allowed for the visualization of the entire small bowel. This study aimed to investigate the clinicopathological features of small bowel malignant tumors diagnosed by VCE and double-balloon enteroscopy (DBE) in a single tertiary center.

Methods: We retrospectively analyzed VCE and DBE findings from Korea University Guro Hospital from January 2010 through September 2018.

Results: A total of 510 VCE and 126 DBE examinations were performed in 438 patients. Small bowel malignancies were diagnosed in 28 patients (15 males; mean age, 61.0 years; range, 42 to 81 years). Among them, 8 had lymphoma, 8 had primary adenocarcinoma, 7 had gastrointestinal stromal tumor (GIST) and 5 had metastatic cancer. Abdominal pain and obstructive symptoms were the most common findings in metastatic cancers $(4 / 5,80 \%)$. On the other hand, obscure gastrointestinal bleeding was the most common symptom of GIST $(6 / 7,85.7 \%)$ and adenocarcinoma $(3 / 8,37.5 \%)$.

Conclusions: Approximately 6\% of the patients who underwent either VCE or DBE were diagnosed with small bowel malignancy. These findings demonstrated the different clinical characteristics among small bowel malignancies and merit further study. Clin Endosc 2021;54:85-91

Key Words: Capsule endoscopy; Endoscopy; Neoplasms; Small intestine

\section{INTRODUCTION}

Even though the small intestine makes up $75 \%$ of the length of the digestive tract, small bowel malignancies are rare, accounting for less than $5 \%$ of gastrointestinal cancers. Annually, about 10,470 new patients are diagnosed with small bowel

Received: February 12, 2020 Revised: June 19, 2020

Accepted: June 20,2020

Correspondence: Beom Jae Lee

Division of Gastroenterology, Department of Internal Medicine, Korea University Guro Hospital, Korea University, College of Medicine, 148 Gurodong-ro, Gurogu, Seoul 08308, Korea

Tel: +82-2-2626-1114, Fax: +82-2-2001-8360, E-mail: L85210@korea.ac.kr ORCID: https://orcid.org/0000-0003-2449-4968

(c) This is an Open Access article distributed under the terms of the Creative Commons Attribution Non-Commercial License (http://creativecommons.org/ licenses/by-nc/3.0) which permits unrestricted non-commercial use, distribution, and reproduction in any medium, provided the original work is properly cited. malignancies, while 1,450 patients die of them. ${ }^{1-4}$ Owing to its nonspecific and ambiguous symptoms, small bowel malignancies are difficult to diagnose and may progress without symptoms. ${ }^{5}$

Various types of malignant tumors occur in the small bowel, including adenocarcinoma, gastrointestinal stromal tumors (GISTs), neuroendocrine tumors (NETs), and lymphoma. ${ }^{2}$ In the past, diagnosis of small bowel malignancies in the early stages was difficult due to limitations of tools. However, the introduction of double-balloon enteroscopy (DBE) has enabled the full evaluation of the entire small bowel, which dramatically improved the diagnosis and treatment of small bowel lesions, including obscure gastrointestinal bleeding and small bowel tumors. ${ }^{5-12}$ Nowadays, video capsule endoscopy (VCE), DBE, and abdominal computed tomography (CT) are used to diagnose small bowel malignancy. Each tool has its advantages and disadvantages. 
VCE is widely used to diagnose small bowel diseases because it is non-invasive and relatively easy to perform. VCE allows for visualization of the small bowel mucosa and its abnormalities on video. ${ }^{7,13,14}$

DBE not only enables full-length visual inspection of the small bowel, but also allows for direct intervention in the deep portions of the jejunum and ileum. It is therefore the most innovative diagnostic tool for small bowel diseases. DBE yields a high rate of successful diagnosis, but it has some limitations, including abdominal pain, side effects from anesthesia or endoscopy, and large economic burden., ${ }^{5,910,12,15,16}$

There is no single best way to diagnose small bowel disease because the currently available tools complement each other. Therefore, the choice of tool for evaluation should be made according to individual patient complaints and conditions. ${ }^{7,12,17}$

In this study, we retrospectively analyzed the clinicopathological features of small bowel malignant tumors that were diagnosed with VCE, DBE, or abdominal CT in a single tertiary center.

\section{MATERIALS AND METHODS}

\section{Patients}

Between January 2010 and September 2018, 438 patients underwent VCE or DBE at Guro Hospital, Korea University. We retrospectively analyzed the findings of VCE, DBE, and abdominal CT as well as their clinicopathologic characteristics. This study was approved by The Ethics Commission of Korea University, Guro Hospital.

\section{Video capsule endoscopy}

VCE was performed with MiroCam ${ }^{\circledR}$ (IntroMedic, Seoul, Korea). Laxatives were used for bowel preparation and the capsule was ingested 2 hours thereafter. The video was recorded for 12 hours, and the capsule was retrieved 24 hours after ingestion. The recorded videos were then analyzed by two enteroscopists, and the lesions were differentiated into three categories: submucosal, ulceroinfiltrative, and bleeding.

\section{Double-balloon enteroscopy}

DBE was performed using EN-450T5 (Fujinon Toshiba ES Systems, Tokyo, Japan). Patients were sedated with midazolam and propofol intravenously. DBE procedures were performed by a single endoscopist (BJL) and were predominantly indicated for tissue confirmation or therapeutic interventions including hemostasis, polypectomy, or foreign body removal. The insertion route was determined by the location of the lesion as evaluated by abdominal CT or VCE. During DBE, biopsy was performed on all lesions. The lesions were then differentiated into four categories, namely, ulceroinfiltrative, submucosal, ulcerofungating, and ulcers with stricture. Every patient underwent either VCE or DBE, and the choice for first-line diagnostic modality depended on the physician.

\section{Abdominal computed tomography}

Abdominal CT was performed either before or after VCE or DBE. The modality of choice may be non-enhanced, enhanced, or enteroclysis CT, based on the judgment of the physician. In some cases, CT was performed multiple times on one patient.

\section{Analyses}

Patients' clinical features and their disease-specific pathologies were analyzed and compared for VCE, DBE and abdominal CT findings. SPSS 11.5 (SPSS Inc., Chicago, IL, USA) was used for all statistical analyses. Continuous variables are presented as mean \pm standard deviation. Categorical variables were analyzed using the chi-square test or Fisher's exact test. A $p$-value lower than 0.05 was considered statistically significant.

\section{RESULTS}

\section{Frequency of malignant small bowel cancer}

A total of $510 \mathrm{VCE}$ and $126 \mathrm{DBE}$ exams was performed on 438 patients from January 2010 to September 2018 at Korea University Guro Hospital. The mean age was $55.1 \pm 18.1$ years, and 228 patients $(52.1 \%)$ were male. Abdominal pain was the chief complaint in $28.8 \%$ (126) of the subjects; $69.2 \%$ (303) of

Table 1. Clinical Characteristics of Enrolled Patients

\begin{tabular}{lc}
\hline Characteristic & $\boldsymbol{n ( \% )}$ \\
\hline Mean age (SD) & $55.1 \pm 18.1$ \\
Male & $228(52.1)$ \\
Chief complain & \\
Abdominal pain & $126(28.8)$ \\
Obscure gastrointestinal bleeding & $303(69.2)$ \\
Other symptom & a) \\
Video capsule endoscopy & $9(2.1)$ \\
Double-balloon enteroscopy & 510 \\
Abdominal CT & 126 \\
Non-enhanced CT & 1 \\
Enhanced CT & 267 \\
Enteroclysis CT & 77 \\
\hline
\end{tabular}

CT, computed tomography; SD, standard deviation.

a) Nauea, vomiting, diarrhea, abnormal image finding. 
cases were obscure gastrointestinal bleeding and proceeded for evaluation (Table 1). As mentioned, 510 VCEs and 126 DBEs were performed in 438 patients, and some patients underwent the same procedure multiple times. Biopsies were obtained as needed (Fig. 1). Of the 126 DBE cases, 67 were via the anus,
49 via the oral route, and 10 via both routes. Among the abdominal CTs performed, only one was with non-enhanced CT, 267 cases were with enhanced CT, and 77 cases were with enteroclysis CT. The following small bowel malignancies were discovered in 28 patients (15 males; mean age, 61.0 years;

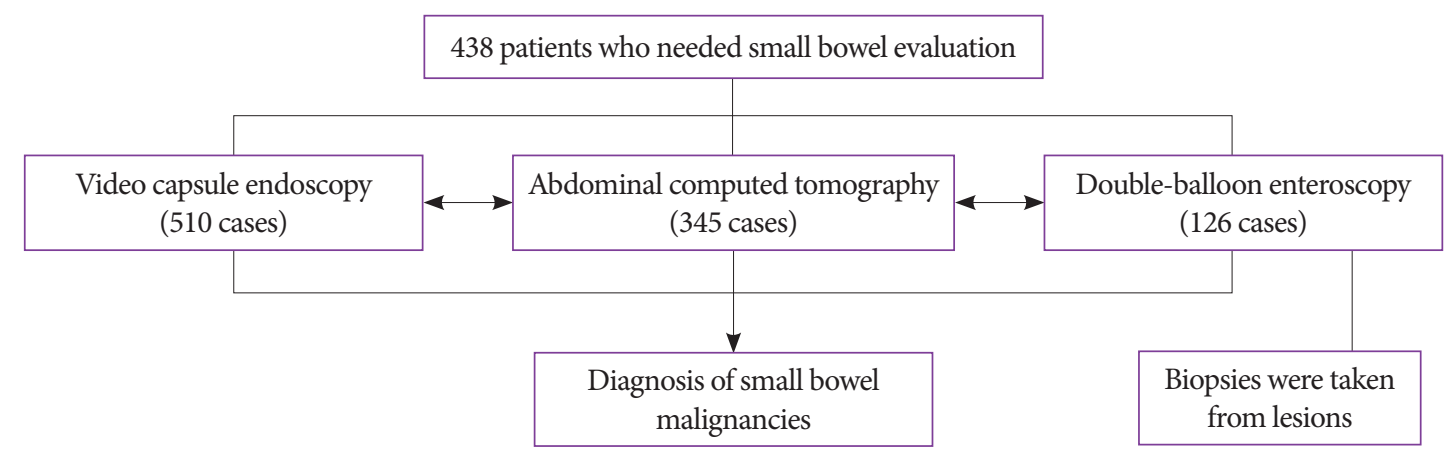

Fig. 1. Study flow chart.

Table 2. Clinical Characteristics, Symptoms and Practical Findings of Small Bowel Malignancy

\begin{tabular}{|c|c|c|c|c|c|}
\hline Characteristic & GIST (7) & Lymphoma (8) & Adenocarcinoma (8) & Metastatic cancer (5) & $p$-value \\
\hline Mean age & $63.5 \pm 11.2$ & $62.3 \pm 11.3$ & $57.3 \pm 9.3$ & $55 \pm 8.6$ & 0.963 \\
\hline $\operatorname{Sex}(M: F)$ & $3: 4$ & $5: 3$ & $4: 4$ & $3: 2$ & \\
\hline Hemoglobin (g/dL) & $8.8 \pm 2.4$ & $11.3 \pm 2.9$ & $9.8 \pm 2.3$ & $9.6 \pm 3.3$ & 0.357 \\
\hline \multicolumn{6}{|l|}{ Symptoms and sign } \\
\hline Obscure GI bleeding & 6 & 2 & 3 & 0 & \\
\hline Abdominal pain and obstructive symptoms & 0 & 2 & 2 & 4 & \\
\hline No symptoms & 0 & 4 & 3 & 1 & \\
\hline Other symptoms & 1 & 0 & 0 & 0 & \\
\hline \multicolumn{6}{|l|}{ CT findings } \\
\hline Eccentric thickening & 0 & 4 & 5 & 2 & \\
\hline Concentric thickening & 0 & 1 & 2 & 3 & \\
\hline Mass formation & 7 & $2^{\text {a) }}$ & 1 & 0 & \\
\hline Unrevealing study & 0 & 1 & 0 & 0 & \\
\hline \multicolumn{6}{|l|}{ DBE findings } \\
\hline Ulceroinfiltrative & 0 & 6 & 3 & 2 & \\
\hline Submucosal mass & 4 & 0 & 0 & 0 & \\
\hline Ulcerofungating & 0 & 0 & 4 & 0 & \\
\hline Ulcer with stricture & 0 & 2 & 1 & 3 & \\
\hline Not done & 3 & 0 & 0 & 0 & \\
\hline \multicolumn{6}{|l|}{ Capsule endoscopy } \\
\hline Submucosal lesions & 2 & 0 & 1 & 0 & \\
\hline Ulceroinfiltrative & 1 & 1 & 2 & 0 & \\
\hline Bleeding & 0 & 0 & 0 & 2 & \\
\hline Not done & 4 & 7 & 5 & 3 & \\
\hline
\end{tabular}

CT, computed tomography; DBE, double-balloon enteroscopy; GI, gastrointestinal; GIST, gastrointestinal stromal tumor.

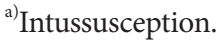


range, 42 to 81 years): 8 lymphomas, 8 primary adenocarcinomas, 7 GISTs, and 5 metastatic cancers (Table 2). Among the 8 cases of lymphoma, 5 were diffuse large B-cell lymphoma, 2 were mucosa-associated lymphoid tissue lymphoma (MALT lymphoma), and 1 was peripheral T-cell lymphoma.

\section{Clinical symptoms of small bowel malignancy}

Among the patients diagnosed with adenocarcinoma, 3 were asymptomatic, 3 had obscure gastrointestinal bleeding, and 2 had abdominal pain and obstructive symptoms. Among those with lymphoma, 4 were asymptomatic, 2 had obscure bleeding, and 2 had abdominal pain and obstructive symptoms. Six of the 7 patients diagnosed with GIST had obscure gastrointestinal bleeding and received small bowel evaluation. Four of the 5 patients with metastatic cancer had abdominal pain and obstructive symptoms (Table 2). Abdominal pain and obstructive symptoms were the most common findings in metastatic cancers $(4 / 5,80 \%)$. Obscure gastrointestinal bleeding was the most common symptom of GIST (85.7\%) and adenocarcinoma (37.5\%).

\section{Video capsule endoscopy and double-balloon enteroscopy findings}

Abdominal CT scans were performed before or after VCE or DBE (Table 2). Nine of 28 patients who were diagnosed with malignancy underwent $\mathrm{VCE}$ and 25 underwent DBE.

VCE was performed on 3 out of 7 GIST patients; 2 showed submucosal tumors and 1 showed ulceroinfiltrative lesions.
Out of 8 lymphoma patients, VCE was performed on 1 patient who showed ulceroinfiltrative lesions. In the case of adenocarcinomas, VCE was performed on 3 patients, which revealed submucosal lesions in 1 and ulceroinfiltrative lesions in 2 patients. Two patients with metastatic cancer underwent VCE, and both involved bleeding lesions.

In the case of GISTs, $57.1 \%$ (4/7) patients with DBE showed submucosal tumors and $42.9 \%$ (3/7) did not undergo DBE. Six of 8 patients with lymphoma showed ulceroinfiltrative lesions and 2 showed ulcers with stricture. Among the 8 cases of adenocarcinoma, ulcerofungating lesions were the most common finding (4/8), followed by ulceroinfiltrative lesions (3/8) and ulcers with stricture $(1 / 8)$. Among the 5 cases of metastatic cancer, $60 \%$ (3/5) showed ulcers with stricture and 40\% (2/5) showed ulceroinfiltrative lesions. The main DBE findings are shown in Fig. 2.

\section{Pathologic finding of double-balloon enteroscopy}

DBEs were performed depending on patient symptoms and the observations from VCE or abdominal CT. The presence of ulceroinfiltrative, ulcerofungating or stricture lesions indicated a biopsy for confirmation of pathologic findings. Biopsy was not performed on submucosal mass. There was a total of 11 cases of ulceroinfiltrative lesions and the final diagnoses of 9 of them were coherent with the pathologic findings of their endoscopic biopsies (Table 3). Three out of 4 were coherent in the case of strictures, and 4 out of 6 in the case of ulcerofungating lesions.

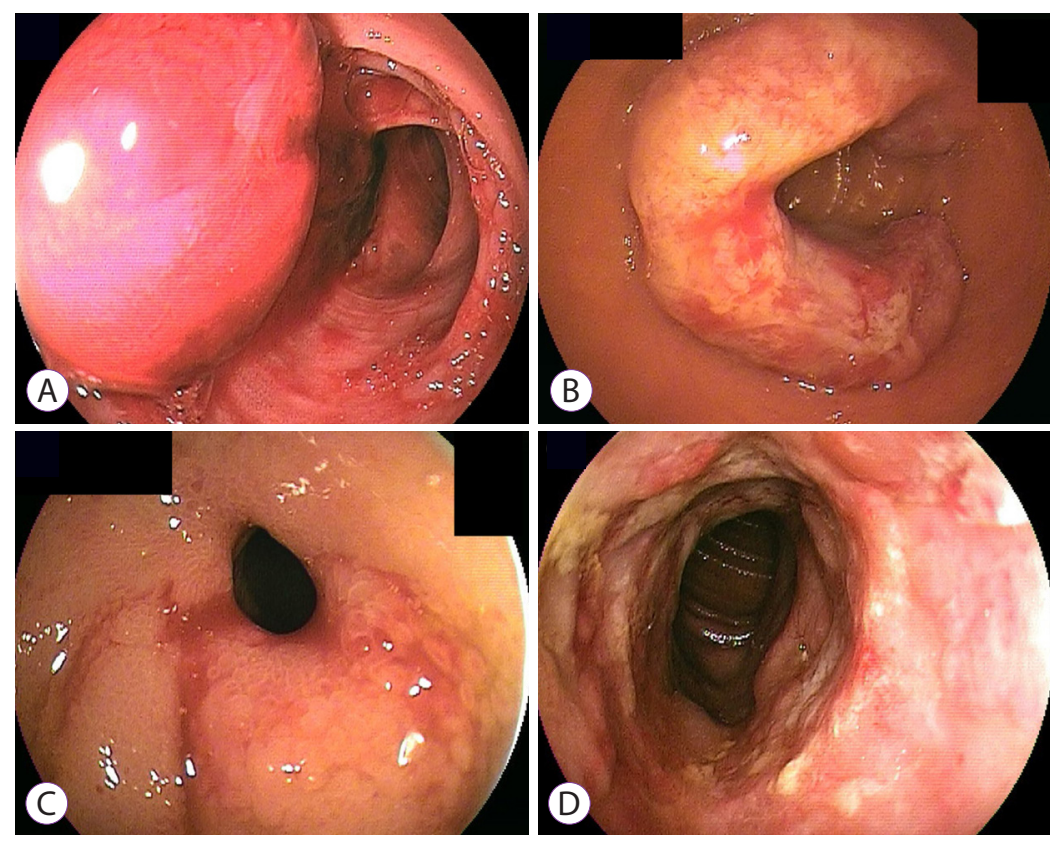

Fig. 2. Representative double-balloon enteroscopy findings. (A) Submucosal mass (B) Ulcerofungating lesions (C) Ulcer with stricture (D) Ulceroinfiltrative lesions. 
Table 3. Concordance Rate between Enteroscopic Biopsies and Final Pathologic Results

\begin{tabular}{lc}
\hline & Concordance rate (\%) \\
\hline Ulceroinfiltrative lesion & $9 / 11(91.8 \%)$ \\
Lymphoma & $6 / 6$ \\
Adenocarcinoma & $1 / 3$ \\
Metastatic cancer & $2 / 2$ \\
Stricture lesion & $3 / 4(75.0 \%)$ \\
Lymphoma & $1 / 2$ \\
Adenocarcinoma & $1 / 1$ \\
Metastatic cancer & $1 / 1$ \\
Ulcerofungating lesion & $4 / 6(66.7 \%)$ \\
Adenocarcinoma & $3 / 4$ \\
Metastatic cancer & $1 / 2$ \\
\hline
\end{tabular}

\section{Locations of small bowel malignancy}

Tumor location varied depending on subtype (Table 4). Tumor location was confirmed through abdominal CT. In cases where abdominal CT was not performed, jejunum- and ileum-located tumors were differentiated depending on the location of enteroscopic findings; close to the mouth or close to the anus. Among the 8 cases of adenocarcinoma, 2 occurred in the duodenum, 5 in the jejunum, and 1 in the ileum. Among the 7 cases of GIST, 28.6\% (2/7) occurred in the ileum and 71.4\% $(5 / 7)$ in the jejunum. Among the 5 cases of metastatic cancer, $80 \%(4 / 5)$ occurred in the jejunum and 20\% (1/5) occurred in the ileum. However, all 8 cases of lymphoma occurred in the ileum. When the 4 subtypes were compared, the locations of tumor differed significantly $(p=0.002)$.

\section{Clinical courses}

Tissue biopsies were done for all ulcerative epithelial lesions. The misdiagnosis rate was $10 \%(2 / 21)$ in cases of ulcerative lesions, and this was also observed in 2 cases of MALT lymphoma. Of the 28 patients diagnosed with small bowel malignancy, $64.2 \%$ underwent small bowel resection. All patients with malignant lymphoma and GIST underwent small bowel resection. Only 3 cases of adenocarcinoma underwent small bowel resection and all metastatic cancers were treated conservatively.

\section{DISCUSSION}

Small bowel malignancy is challenging to diagnose because the small bowel is difficult to evaluate. Diagnosis is further challenged owing to the nonspecific symptoms and, sometimes, the absence of symptoms during malignancy progression. Diagnosis and treatment are therefore usually delayed, and the consequences tend to be serious. ${ }^{18-20}$ Abdominal CT is a valuable tool for diagnosing patients with small bowel malignancies, but there are many cases in which abdominal CT cannot provide useful information. Risk factor analyses may facilitate the diagnostic process, but the risk factors for small bowel cancer are difficult to predict because of its rarity and the various types of tumors that may be involved. ${ }^{7}$

After development of VCE in 2000, the entire small bowel and its lumen could be fully evaluated. VCE is relatively non-invasive, and is often used when small bowel malignancy is suspected following abdominal CT. However, VCE has its limitations, including false positives and false negatives as well as limited options for immediate action when malignancy is found. ${ }^{9,10}$

Since the introduction of DBE in the early 2000s, a large amount of data has proven its diagnostic value for patients with small bowel pathologies. Like VCE, DBE allows full examination of the entire small bowel, which can help identify pathologies such as bleeding, strictures, and tumors. However, DBE can also be used for a variety of therapeutic interventions, including hemostasis, polypectomy, endoscopic mucosal resection, balloon dilatation, and stent insertion. ${ }^{7,12,17}$

Abdominal CT, VCE, and DBE are not meant to be used exclusively, but to complement each other depending on circumstances such as patient condition. ${ }^{7,12}$ According to a guideline for enteroscopy published in Japan in 2017, VCE is recommended as a first-line diagnostic modality for obscure gastrointestinal bleeding, but is contraindicated for obstructive

Table 4. Tumor Subtypes Found in the Small Bowel Varies depending on the Location

\begin{tabular}{lccccc}
\hline & GIST & Lymphoma & Adenocarcinoma & Metastatic cancer & 0 \\
\hline Duodenum & 0 & 0 & $2(25 \%)$ & $4(80 \%)$ & 0.002 \\
Jejunum & $5(71.4 \%)$ & 0 & $5(62.5 \%)$ & $1(20 \%)$ \\
\hline Ileum & $2(28.6 \%)$ & $8(100 \%)$ & $1(12.5 \%)$ & \\
\hline
\end{tabular}

GIST, gastrointestinal stromal tumor. 
symptoms. It also suggests CT, magnetic resonance imaging, and enteroclysis as first diagnostic modalities for large tumors in the small intestine, and VCE for easier observation of small neoplasms or flat lesions. Thus, when evaluating the small bowel, it is necessary to perform the appropriate modalities depending on the different suspected conditions. ${ }^{21}$

Abdominal CT and VCE were used as screening examinations for small bowel diseases, while DBE was used for tissue biopsy and therapeutic interventions. For example, after achieving a diagnosis through abdominal CT and VCE, DBE would be performed if a biopsy or therapeutic intervention is needed. As DBE is more invasive than abdominal CT and VCE, it is unfeasible to be performed in every patient. Moreover, the requirement for sedation to reduce abdominal discomfort and pain may cause side effects such as desaturation and allergic reactions. There is also the possibility of endoscopic side effects, including bleeding and perforation. Finally, financial burden is another concern in patients undergoing endoscopy. For these reasons, DBE was only performed on selected patients who needed it the most. ${ }^{7,12,17}$

In our study, 28 patients were diagnosed with small bowel malignancy. The most common signs and symptoms were obscure bleeding $(11 / 28,39.3 \%)$, as well as abdominal pain and weight loss. The clinical signs varied depending on malignancy subtype. In cases of lymphoma, abdominal pain, including obstructive symptoms, was most common (4/8, 50\%), whereas obscure bleeding was most common in cases of GIST (6/7, $85.7 \%)$. The presenting symptoms or clinical signs may be explained by the endoscopic features of each tumor type. For example, malignant lymphomas harbor encircling and ulcerative lesions, whereas a mass with ulceration is the major finding of GIST.

In a previous study, abnormal imaging findings were the most common clinicopathologic features of small bowel tumors, and the most common symptoms were bleeding and abdominal pain, including obstructive symptoms. ${ }^{5}$ In the present study, 11 subjects (39.3\%) showed bleeding, and 8 (28.6\%) showed abdominal pain and obstructive symptoms. Moreover, all 438 patients in this study underwent abdominal CT, and 27 of the 28 patients (96.4\%) who were diagnosed with small bowel malignancy had positive CT findings, including heterogeneous wall thickening or masses (in all cases of GIST, adenocarcinoma, and metastatic cancer). However, in terms of lymphomas, 2 cases of MALT lymphoma showed positive endoscopic findings despite negative CT results. Abdominal CTs showed high sensitivity for diagnosing small bowel malignancy, but a positive finding itself cannot diagnose the exact subtype. As exemplified by the 2 cases of lymphoma with negative abdominal CT findings, additional examinations with DBE or VCE are required regardless of CT findings.
In the present study, tumor location significantly differed according to the type of malignancy. GIST was mainly located in the proximal small bowel, whereas malignant lymphoma was located at the distal portion of the duodenum. Horie et al. reported in 2019 that they diagnosed 16 epithelial tumors, 22 small bowel lymphomas, and 6 GISTs in 1,328 patients with DBEs. ${ }^{22}$ However, they failed to show statistical significance between tumor locations in the small bowel and the types of cancer. In this study, small bowel tumors were classified into 4 types; $71.4 \%$ of GIST was observed in the jejunum, $100 \%$ of lymphoma in the ileum, $62.5 \%$ of adenocarcinoma in the jejunum, and $80 \%$ of metastatic cancer in the jejunum. The study period was shorter and the number of subjects was smaller than those in Horie et al., but it showed statistically significant results with a $p$-value of $0.002{ }^{22}$ Therefore, abdominal CT findings in a specific anatomical location may be helpful in predicting the properties of small bowel malignancy and in planning for future treatment approaches.

There was no case of NET in our study, and this was in line with studies conducted in other Asian countries. ${ }^{8,15,23}$ In a Korean multicenter study, the most common small bowel tumors were GIST ( $n=29,25.9 \%)$, lymphoma ( $n=18,16.1 \%)$, and adenocarcinoma $(n=14,12.5 \%) .{ }^{8}$ In a Taiwanese study on 1,070 cases of DBE, the most common type was lymphoma ( $n=20,29.0 \%)$, followed by GIST ( $n=19,27.5 \%)$, adenocarcinoma $(n=18,26.1 \%)$, metastatic cancer $(n=10,14.5 \%)$, and only a few cases of NET $(n=2,0.9 \%) .{ }^{15}$ In contrast, NET was the most common type of small bowel malignancy in Western studies. Cangemi et al. showed that in 1,652 DBEs, the most common lesions were NET (19.4\%), GIST (7.5\%), and lymphoma $(7.5 \%)^{5}$

Regarding the prevalence of NET, a study from the United States showed that out of 25,531 cases of gastrointestinal NET, $38 \%$ occurred in the small bowel and 34\% occurred in the rectum. ${ }^{24}$ In contrast, a Korean study showed that a majority of the cases of gastrointestinal NET were detected in the rectum (48\%), $15 \%$ in the stomach, and $9 \%$ in the pancreas. ${ }^{18}$ The reasons for this epidemiological difference are unclear. Differences in ethnicity and the environment of clinical practice between Western and Asian countries might influence the location of gastrointestinal NET. Further research is therefore needed.

One limitation of this study is its retrospective-nature, which posed a possibility of bias. In addition, the small number of small bowel malignancy cases (28 patients) led to statistically nonsignificant results, although a trend could be drawn. However, we focused on the malignant cancer patients exclusively, and we had 438 patients who received small bowel evaluation, which is not few when compared to the other studies.

In conclusion, adenocarcinoma, malignant lymphoma, and 
GISTs were observed to be the most common small bowel malignant tumors. In our study, 28 of 438 patients who underwent VCE or DBE were diagnosed with small bowel malignancy, and tumor location significantly differed by tumor type. These findings elucidated the different clinical characteristics of each type of small bowel malignancy and hence merit further study.

Conflicts of Interest

The authors have no potential conflicts of interest.

Funding

None.

Author Contributions

Conceptualization: Beom Jae Lee

Data curation: BJL, Seung Han Kim, Moon Kyung Joo, Hyo Jung Kim, Jong-Jae Park

Formal analysis: Ah Young Yoo, Won Shik Kim, Seong Min Kim

Methodology: AYY, BJL

Project administration: BJL

Supervision: BJL

Visualization: BJL, MKJ, JJP

Writing-original draft: AYY, BJL

Writing-review\&editing: AYY, BJL

\section{ORCID}

Ah Young Yoo:

Beom Jae Lee:

Won Shik Kim:

Seong Min Kim:

Seung Han Kim:

Moon Kyung Joo:

Hyo Jung Kim:

Jong-Jae Park: https://orcid.org/0000-0002-3474-2016 https://orcid.org/0000-0003-2449-4968 https://orcid.org/0000-0003-4431-6472 https://orcid.org/0000-0003-4438-2955 https://orcid.org/0000-0001-9247-9175 https://orcid.org/0000-0001-6050-3695 https://orcid.org/0000-0003-3284-3793 https://orcid.org/0000-0002-4642-5405

\section{REFERENCES}

1. de Franchis R, Rondonotti E, Abbiati C, Beccari G, Signorelli C. Small bowel malignancy. Gastrointest Endosc Clin N Am 2004;14:139-148.

2. Pan SY, Morrison H. Epidemiology of cancer of the small intestine. World J Gastrointest Oncol 2011;3:33-42.

3. Schottenfeld D, Beebe-Dimmer JL, Vigneau FD. The epidemiology and pathogenesis of neoplasia in the small intestine. Ann Epidemiol 2009;19:58-69.

4. Schwartz GD, Barkin JS. Small bowel tumors. Gastrointest Endosc Clin N Am 2006;16:267-275.
5. Cangemi DJ, Patel MK, Gomez V, Cangemi JR, Stark ME, Lukens FJ. Small bowel tumors discovered during double-balloon enteroscopy: analysis of a large prospectively collected single-center database. J Clin Gastroenterol 2013;47:769-772.

6. Cobrin GM, Pittman RH, Lewis BS. Increased diagnostic yield of small bowel tumors with capsule endoscopy. Cancer 2006;107:22-27.

7. Kim JH, Moon W. Optimal diagnostic approaches for patients with suspected small bowel disease. Clin Endosc 2016;49:364-369.

8. Lee BI, Choi H, Choi KY, et al. Clinical characteristics of small bowel tumors diagnosed by double-balloon endoscopy: KASID multi-center study. Dig Dis Sci 2011;56:2920-2927.

9. Postgate A, Despott E, Burling D, et al. Significant small-bowel lesions detected by alternative diagnostic modalities after negative capsule endoscopy. Gastrointest Endosc 2008;68:1209-1214.

10. Ross A, Mehdizadeh S, Tokar J, et al. Double balloon enteroscopy detects small bowel mass lesions missed by capsule endoscopy. Dig Dis Sci 2008;53:2140-2143.

11. Urbain D, De Looze D, Demedts I, et al. Video capsule endoscopy in small-bowel malignancy: a multicenter Belgian study. Endoscopy 2006;38:408-411.

12. Yen HH, Chang CW, Chou JW, Wei SC. Balloon-assisted enteroscopy and capsule endoscopy in suspected small bowel Crohn's disease. Clin Endosc 2017;50:417-423.

13. Hosoe N, Takabayashi K, Ogata H, Kanai T. Capsule endoscopy for small-intestinal disorders: current status. Dig Endosc 2019;31:498-507.

14. Valero M, Bravo-Velez G, Oleas R, et al. Capsule endoscopy in refractory diarrhea-predominant irritable bowel syndrome and functional abdominal pain. Clin Endosc 2018;51:570-575.

15. Chung CS, Tai CM, Huang TY, et al. Small bowel tumors: a digestive endoscopy society of Taiwan (DEST) multicenter enteroscopy-based epidemiologic study. J Formos Med Assoc 2018;117:705-710.

16. Hermans C, Stronkhorst A, Tjhie-Wensing A, et al. Double-balloon endoscopy in overt and occult small bowel bleeding: results, complications, and correlation with prior videocapsule endoscopy in a tertiary referral center. Clin Endosc 2017;50:69-75.

17. Kim J. Training in endoscopy: enteroscopy. Clin Endosc 2017;50:328333.

18. Guo X, Mao Z, Su D, Jiang Z, Bai L. The clinical pathological features, diagnosis, treatment and prognosis of small intestine primary malignant tumors. Med Oncol 2014;31:913.

19. Kim CH, Kye BH, Lee JI, et al. Clinicopathological features of primary jejunoileal tumors. J Korean Soc Coloproctol 2010;26:334-338.

20. Yang YS, Huang QY, Wang WF, Sun G, Peng LH. Primary jejunoileal neoplasmas: a review of 60 cases. World J Gastroenterol 2003;9:862-864.

21. Yamamoto $\mathrm{H}$, Ogata $\mathrm{H}$, Matsumoto $\mathrm{T}$, et al. Clinical practice guideline for enteroscopy. Dig Endosc 2017;29:519-546.

22. Horie T, Hosoe N, Takabayashi K, et al. Endoscopic characteristics of small intestinal malignant tumors observed by balloon-assisted enteroscopy. World J Gastrointest Endosc 2019;11:373-382.

23. Chen EY, Vaccaro GM. Small bowel adenocarcinoma. Clin Colon Rectal Surg 2018;31:267-277.

24. Mocellin S, Nitti D. Gastrointestinal carcinoid: epidemiological and survival evidence from a large population-based study $(\mathrm{n}=25531)$. Ann Oncol 2013;24:3040-3044. 